\title{
A Polynomial Model of Patient-specific Breathing Effort During Controlled Mechanical Ventilation
}

\author{
Daniel P Redmond, Paul D Docherty, Yeong Shiong Chiew and J Geoffrey Chase
}

\begin{abstract}
Patient breathing efforts occurring during controlled ventilation causes perturbations in pressure data, which cause erroneous parameter estimation in conventional models of respiratory mechanics. A polynomial model of patient effort can be used to capture breath-specific effort and underlying lung condition. An iterative multiple linear regression is used to identify the model in clinical volume controlled data. The polynomial model has lower fitting error and more stable estimates of respiratory elastance and resistance in the presence of patient effort than the conventional single compartment model. However, the polynomial model can converge to poor parameter estimation when patient efforts occur very early in the breath, or for long duration. The model of patient effort can provide clinical benefits by providing accurate respiratory mechanics estimation and monitoring of breath-to-breath patient effort, which can be used by clinicians to guide treatment.
\end{abstract}

\section{INTRODUCTION}

Respiratory mechanics monitoring of critically ill patients receiving mechanical ventilation can be used to guide treatment and monitor changes in patient condition [1], [2]. The underlying respiratory mechanics of a patient, indicative of the lung condition, can be masked by any level of patient breathing effort [3]. Models that take into account the patient effort could be used to provide a more stable estimate of the slowly changing lung condition changes, reflecting how the recruitment status or airway obstructions in the lungs do not change greatly from breath to breath [4]. Such a model can account for the variation in pressure waveforms by the patient's respiratory effort changing from breath to breath.

Patients breathing efforts on top of ventilator support can cause large perturbations in respiratory data [5]. In volume control ventilation, this effort appears as a drop in the airway pressure during inspiration. Fig. 1b. shows how the single compartment model [6] fits poorly to data where the patient breathing effort has a large effect on the airway pressure profile. A poorly-fitted model will produce erroneous parameter identification and it is not suitable for clinical application, though care needs to be taken with additional parameterising of the model, as this can cause parameter trade-off, and limits the accuracy of the model [7]. Thus, a model that can capture patient and breath-specific effort is needed. This paper presents a model of patient breathing effort and the process to identify the model parameters using clinical data.

D P Redmond, P D Docherty, Y S Chiew, and J G Chase are with the Department of Mechanical Engineering, University of Canterbury, Christchurch, New Zealand (corresponding author D Redmond, email: daniel.redmond@pg.canterbury.ac.nz).
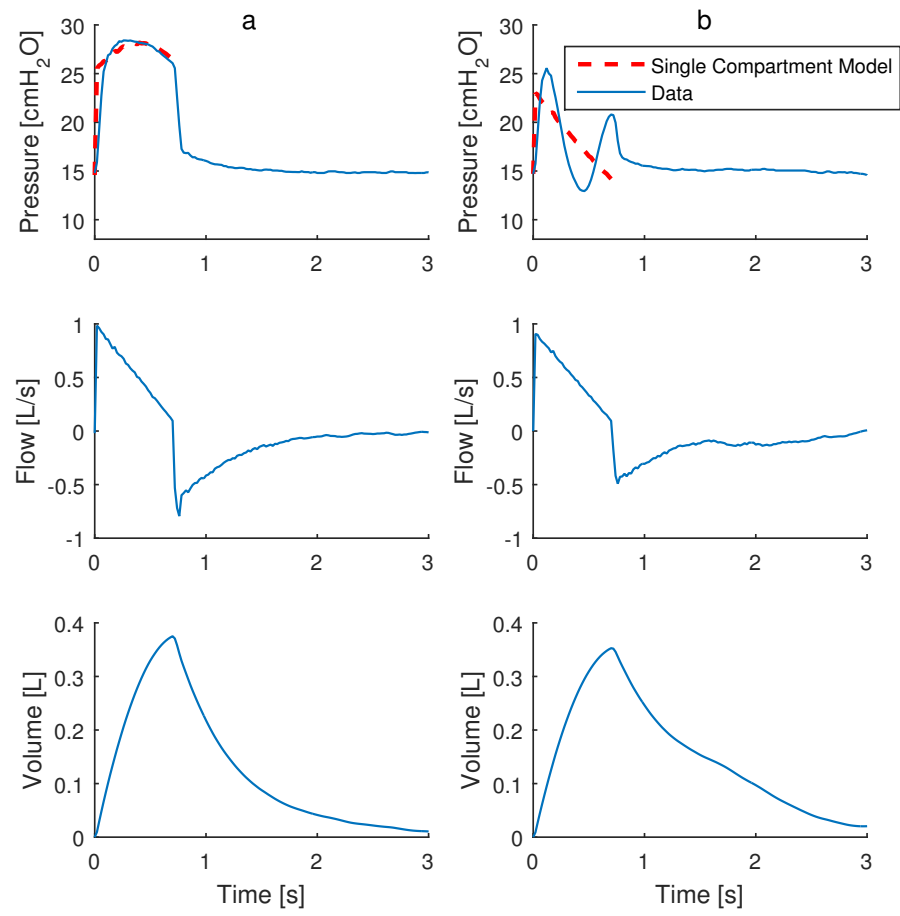

Fig. 1. Part a shows that a linear combination of flow and volume provides a good fitting of the single compartment model to airway pressure from clinical data where there is no patient breathing effort. Part b side shows a linear combination of flow and volume is a poor fit to a pressure waveform that is significantly modified by patient breathing effort

\section{Methods}

To model the patient's respiratory mechanics when patient breathing efforts are present, an assumed shape of patient effort was used. This shape models the effect of reversetriggering observed during mechanical ventilation volume control mode [5], [8]. The conventional single compartment lung model [6] is modified to include a patient effort function $P_{e}(t)$. The airway pressure can be represented as follows during inspiration:

$$
P_{a w}(t)=E \times V(t)+R \times Q(t)+P_{0}+P_{e}(t)
$$

$P_{a w}$ is airway pressure $\left(\mathrm{cmH}_{2} \mathrm{O}\right), E$ is respiratory system elastance $\left(\mathrm{cmH}_{2} \mathrm{O} / \mathrm{L}\right), R$ is respiratory system resistance $\left(\mathrm{cmH}_{2} \mathrm{O} \cdot \mathrm{s} / \mathrm{L}\right), V$ is inspired volume (L), $Q$ is inspiratory flow $(\mathrm{L} / \mathrm{s})$ and $P_{0}$ is offset pressure. $P_{e}$ is the perturbation in airway pressure caused by the patients respiratory effort during volume control ventilation. A quadratic shape is assumed for 

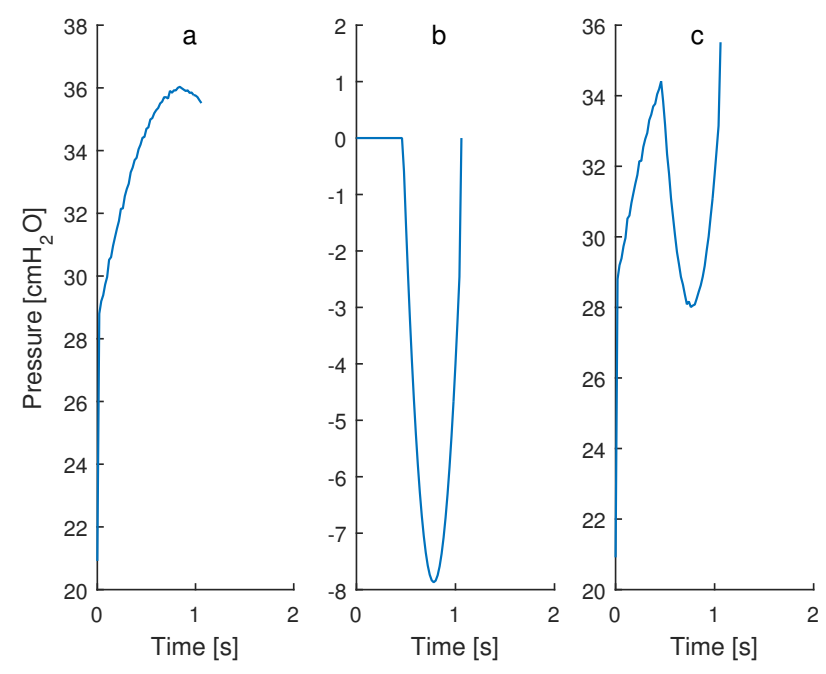

Fig. 2. Contributions of pressures from elastance and resistance (a), and the pressure from the patient effort (b) to make the overall pressure waveform (c).

the patient effort based on observation of pressure waveforms from clinical data as shown in Fig. 1b. $P_{e}(t)$ is modelled below:

$$
P_{e}(t)= \begin{cases}0 & t<t_{s} \\ a t^{2}+b t+c & t_{s} \leq t<t_{f} \\ 0 & t \geq t_{f}\end{cases}
$$

$a, b$ and $c$ define the shape and position of the quadratic effort function. $t_{s}$ and $t_{f}$ come from the roots of the quadratic function, and indicate the start and finish respectively of the patient breathing effort. Equation (2) is only valid where the quadratic function has real valued roots during the time of inspiration. Additionally, $a$ should always be positive so that the patient effort is concave up parabola. Fig. 2. shows how the parabolic shaped patient effort(b) is added to the elastic and resistive pressures (a) to obtain a modified airway pressure curve (c). If $t_{s}$ and $t_{f}$ are known, the airway pressure is a linear combination of volume, flow, time and time squared. This enables the model parameters $E, R, a, b$, and $c$ to be identified by multiple linear regression [9]. The model can then be formulated as follows:

$$
\left[\begin{array}{ccccc}
V\left(t_{0}\right) & Q\left(t_{0}\right) & 0 & 0 & 0 \\
\vdots & \vdots & \vdots & \vdots & \vdots \\
V\left(t_{s}\right) & Q\left(t_{s}\right) & t_{s}^{2} & t_{s} & 1 \\
\vdots & \vdots & \vdots & \vdots & \vdots \\
V(t) & Q(t) & t^{2} & t & 1 \\
\vdots & \vdots & \vdots & \vdots & \vdots \\
V\left(t_{f}\right) & Q\left(t_{f}\right) & t_{f}^{2} & t_{f} & 1 \\
\vdots & \vdots & \vdots & \vdots & \vdots \\
V(t) & Q(t) & 0 & 0 & 0
\end{array}\right] \times\left[\begin{array}{c}
E \\
R \\
a \\
b \\
c
\end{array}\right]=\left[\begin{array}{c}
P\left(t_{0}\right)-P_{0} \\
\vdots \\
P\left(t_{s}\right)-P_{0} \\
\vdots \\
P(t)-P_{0} \\
\vdots \\
P\left(t_{f}\right)-P_{0} \\
\vdots \\
P(t)-P_{0}
\end{array}\right]
$$

Equation (3) can be solved for the best fit parameter values for $E, R, a, b$, and $c$ using least squares error. In this study an

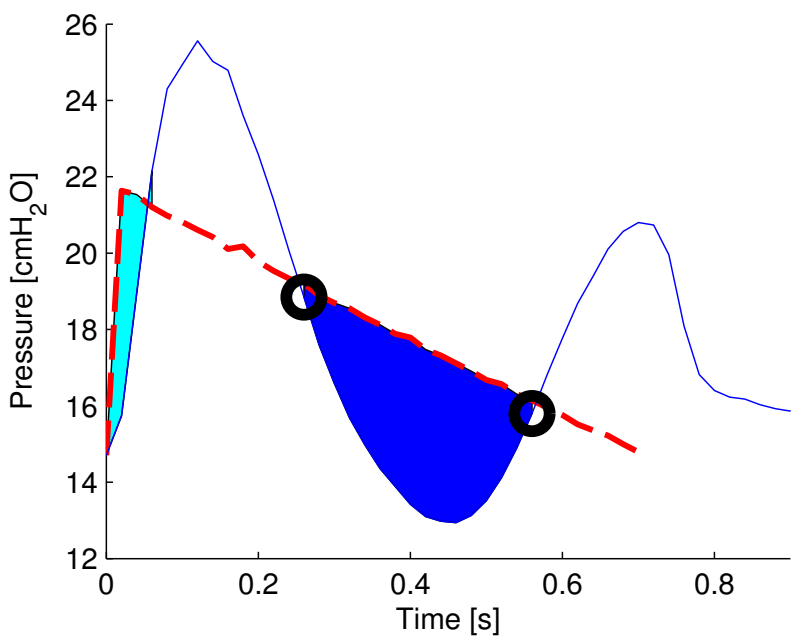

Fig. 3. Finding initial values for $t_{s}$ and $t_{f}$ by fitting the conventional single compartment model. Cyan and blue shaded regions show where $P_{\text {model }}>$ $P_{\text {data }}$, as the blue area is larger, the edges of this region are chosen for initial estimates of $t_{s}$ and $t_{f}$ as shown by the marked circles.

iterative approach is then used [9], where the identified $a, b$, and $c$ are used to find $t_{s}$ and $t_{f}$ from the roots of the quadratic function.

Initial values of $t_{s}$ and $t_{f}$ are required, and must be reasonably close to the global minimum to ensure convergence, otherwise the solution can converge to a local minimum that is not the best fit.

\section{A. Initial value selection}

The initial values for $t_{s}$ and $t_{f}$ are determined by fitting the conventional single compartment lung model using a hierarchical approach [10]. The fitted model will have regions where the modelled pressure is lower than the pressure data. The largest of these regions is likely to be where the patient effort is occurring. This initial point is found by integrating the pressure difference over the regions where $P_{\text {model }}>P_{\text {data }}$. The maximum of these integrated area then provides an initial guess for $t_{s}$ and $t_{f}$ as shown in Fig. 3.

\section{B. Model refinements}

If a patient breathing effort is not present during inspiration, the use of this model can result in convergence to physiologically implausible parameters. To ensure the parameter values are reasonable, and that key features of the airway pressure waveform are modelled by the appropriate parameters, there are a number of checks made to the converged parameter values. If the converged solution fails any of these checks, the model reverts back to the single compartment model, with no patient effort estimation. Both $E$ and $R$, were bounded between 0 and 500. If $a$ is negative, the quadratic effort function becomes concave down and can model the entire inspiration, rather than just the patient effort. Hence $a$ is bounded to positive values. If the roots of the patient effort quadratic function are complex, the model reverts to the single compartment model. 


\section{Quantification of level of patient effort}

By integrating the absolute value of the identified patient effort function, a metric for the size of the patient effort can be gained. This integral needs to be scaled by dividing by tidal volume for that breath so it is proportional to the total breath size. The integral should not be scaled by peak pressure, or level of pressure support, as the effort would be relatively underestimated in patients with high respiratory elastance, and overestimated when patients have low respiratory system elastance.

\section{RESULTS}

Table I shows the distribution of identified parameters and model fitting error across 264 breaths at 5 different levels of positive end-expiratory pressure (PEEP).

Fig. 4. shows three examples of the model fitting for breaths with and without patient effort.

\section{DISCUSSION}

The occurrence of patient effort caused the conventional single compartment model to perform poorly, as expected. The conventional model resulted in high model fitting error, and typically overestimates $R$ and underestimates $E$. An example can be seen in of Figure 4(L), where the conventional model overestimates pressure early in inspiration, where flow is high and volume is low, hence pressure due to resistance dominates. Poor estimates of elastance and resistance are problematic when attempting to guide clinical treatment using respiratory mechanics [1]. The polynomial model presented in this study is capable of capturing patient breathing effort during volume controlled ventilation. By allowing a time period to have a perturbation of measured pressure from modelled pressure, it enables $E$ and $R$ to fit better in the region unaffected by patient effort. In addition, the polynomial parameters were able to provide a unique quantification of breath-specific effort. These efforts observed in the data used for this study vary breath-to-breath, suggesting breathing asynchrony as well as variability. However, the application of these metrics warrants further investigation

The polynomial model can only converge to a solution where the initial estimate for $t_{s}$ and $t_{f}$ are reasonably close to the actual position. $t_{s}$ and $t_{f}$ can only move incrementally from one iteration to the next, as they are found from the roots of the quadratic effort function. Therefore, a robust estimation of initial position is important to ensure convergence. Using the integrals of the differences in modelled pressure and data works well to get $t_{s}$ and $t_{f}$ in the right general area. The polynomial model is designed to capture breath specific effort during volume control ventilation modes, and as such, has only been tested with volume control clinical data. Patient efforts in volume control are seen as perturbations in pressure, as the ventilator is controlling flow, and hence volume. Patient efforts in pressure control modes cause perturbations in flow profiles, and therefore would require a different modelling approach.

This model assumes a parabolic breathing effort profile. A parabola was arbitrarily chosen as it can be described with three parameters, and as it can be easily linearised for solving the least squares problem. However, there is limited physiological basis for picking a parabola, and a similar model could be made using part of a cosine curve or any other function of a similar shape. In this study, the polynomial model is used as a proof of concept to capture breath-specific effort variability. Thus, reformulating the model can equally capture these efforts without parameter trade off.

Patient efforts that occur very early in the breath, or continue for a high proportion of the inspiration time, may cause poor parameter estimations. The Fig. 4(C). shows an example of a relatively early and long patient effort. The model has a relatively low fitting error in this situation, as the region of patient effort has low error, but the model predicts a peak airway pressure $80 \mathrm{cmH}_{2} \mathrm{O}$ when patient effort is removed, with corresponding elastance and resistance of $176 \mathrm{cmH}_{2} \mathrm{O} / \mathrm{L}$ and $-0.55 \mathrm{cmH}_{2} \mathrm{Os} / \mathrm{L}$ respectively. These values are not within a physiologically plausible range, and occur because there is limited data unaffected by patient effort. Data that is unaffected by patient effort is required to get a good estimates of $E$ and $R$, thus pooling breathing cycles or Bayesian analyses could be used to increase model stability [11]

Breaths that do not contain an obvious dip in pressure can sometimes result in a converged solution where elastance and resistance are both close to zero, and the entire shape of the curve is described by a concave down parabola. Obviously this does not reflect the physical cause of the pressure profile, and as such is an inappropriate solution. When this occurs, the model automatically reverted to the conventional single compartment model.

The polynomial model tends to fit poorly in the region close to $t_{s}$. This is due to the sudden start of patient effort in the model, which causes a sharp corner in the modelled pressure profile as can be seen in Fig. 4. A sharp corner is not really going to exist in the pressure data, so the data will always cut inside the model at the corner. This poor fitting could possibly be addressed by using a patient effort function that has a gradient of 0 at $t_{s}$, such as a cosine curve. Implementing a cosine function would require a change of the parameter identification process as it cannot be readily be linearised, as required for multiple linear regression.

This polynomial model has shown promising results but could benefit from future work by changing the method of parameter identification. Multiple linear regression is limited to functions that can be linearised, and the cost function is calculated from equal weightings of all data points. A different method could be developed to suit this situation with a customised cost functions that can more strongly penalize error in certain parts of the breath, and include penalty terms for certain parameter estimations. This would likely remove some of the issues with converging to non-physiological parameter estimates, such as negative resistance and elastance.

The model of patient effort allows the level of patient effort to be quantified breath by breath. Similar metrics are available by using oesophageal pressure or electrical activity of the diaphragm measurements [12]. However, these methods 
TABLE I

MEDIAN [IQR] OF IDENTIFIED MODEL PARAMETERS, ROOT MEAN SQUARED ERROR (RMSE) OF THE MODEL FITTING, AND ESTIMATED PATIENT EFFORT.

\begin{tabular}{l|lllll|l|l} 
PEEP & \multicolumn{1}{c}{$\mathrm{E}$} & \multicolumn{1}{c}{$\mathrm{R}$} & \multicolumn{1}{c}{$\mathrm{a}$} & \multicolumn{1}{c}{$\mathrm{b}$} & \multicolumn{1}{c}{$\mathrm{c}$} & \multicolumn{1}{c}{ RMSE } & \multicolumn{1}{c}{ Effort } \\
\hline 15 & $28.0[25.2-30.5]$ & $8.75[7.38-9.15]$ & $66.9[43.7-95.3]$ & $-95.6[-135--72.5]$ & $24.5[15.9-32.1]$ & $0.82[0.74-0.88]$ & $0.54[0-4.84]$ \\
17 & $35.1[30.4-37.9]$ & $7.42[6.80-8.78]$ & $71.4[58.7-79.4]$ & $-119[-132--98.1]$ & $36.8[28.6-46.7]$ & $0.76[0.72-0.87]$ & $7.51[0.52-11.2]$ \\
19 & $35.3[33.0-40.5]$ & $7.98[6.86-8.76]$ & $58.2[47.0-4250]$ & $-95.9[-265--80.8]$ & $19.9[-0.40-26.6]$ & $0.72[0.67-0.84]$ & $0.54[0-8.12]$ \\
21 & $38.7[35.4-39.7]$ & $8.08[7.73-8.73]$ & $47.3[30.1-4400]$ & $-63.3[-273--47.9]$ & $8.07[-0.42-16.5]$ & $0.78[0.53-0.82]$ & $0.52[0-1.75]$ \\
23 & $43.3[42.4-43.8]$ & $8.12[7.68-8.81]$ & $4276[4095-4407]$ & $-269[-274--258]$ & $-0.50[-0.56--0.45]$ & $0.72[0.29-0.82]$ & $0.24[0-0.53]$
\end{tabular}
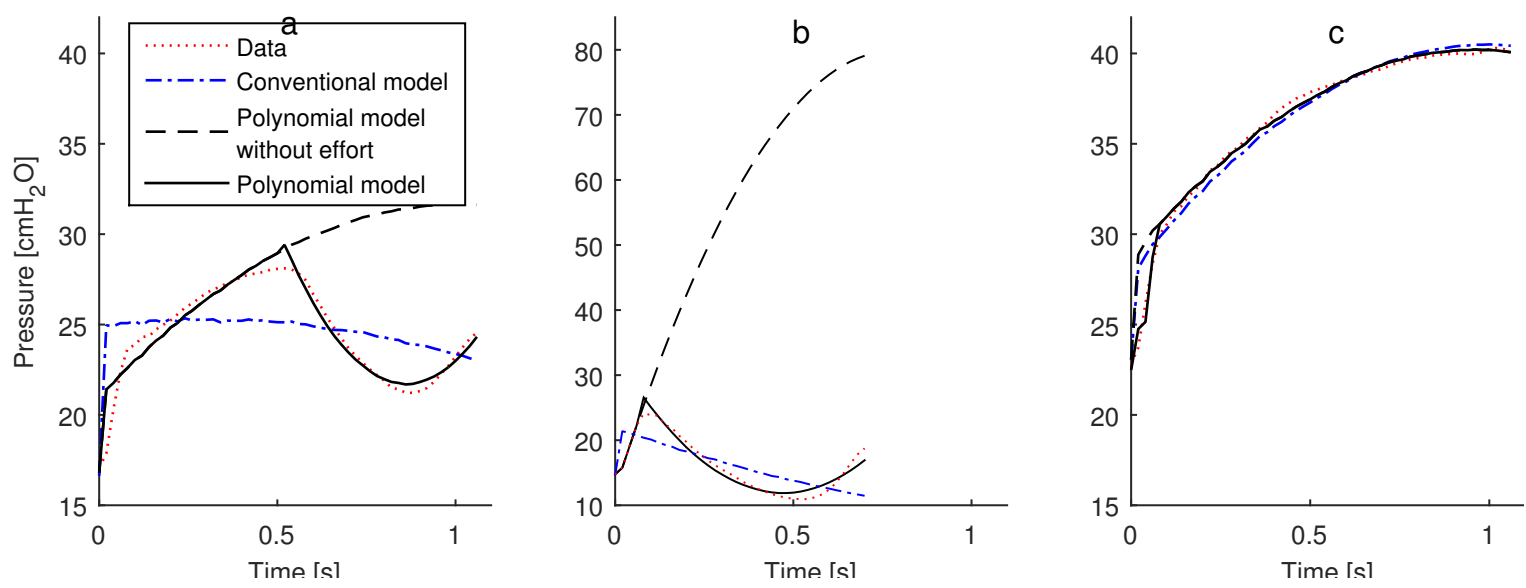

Fig. 4. Example of three model fits. Left panel shows reasonably good fitting of the polynomial model to a breath that has an obvious patient effort in the later part of the breath, the conventional single compartment model performs very poorly in this situation. The middle panel shows a poor fitting of the polynomial model to a breath with a long patient effort. The polynomial model has converged to a solution that predicts a very high peak pressure. The right panel shows a breath with no obvious patient effort, the model has erroneously identified the mismatch between pressure and flow, at the beginning of inspiration, as a patient effort.

require additional equipment that is both more invasive and costly. Monitoring changes in patients breathing efforts during controlled ventilation modes can indicate to clinicians that a mode of ventilation that allows spontaneous breathing may be appropriate. Conversely, the patient efforts may be harmful for other clinical reasons, and increased sedation and muscle relaxants may be appropriate [13]. This model of patient effort allows the changes in patient effort to be monitored over time, different PEEP levels and different ventilator settings.

\section{REFERENCES}

[1] M.-C. Pintado, R. de Pablo, M. Trascasa, J.-M. Milicua, S. Rogero, M. Daguerre, J.-A. Cambronero, I. Arribas, and M. Sánchez-García, "Individualized PEEP setting in subjects with ARDS: a randomized controlled pilot study." Respir. Care, vol. 58, no. 9, pp. 1416-23, Sep. 2013.

[2] Y. S. Chiew, C. G. Pretty, G. M. Shaw, Y. W. Chiew, B. Lambermont, T. Desaive, and J. G. Chase, "Feasibility of titrating PEEP to minimum elastance for mechanically ventilated patients," Pilot Feasibility Stud., vol. 1, pp. 1-10, 2015.

[3] D. Talmor, T. Sarge, A. Malhotra, C. R. O’Donnell, R. Ritz, A. Lisbon, V. Novack, and S. H. Loring, "Mechanical ventilation guided by esophageal pressure in acute lung injury," N. Engl. J. Med., vol. 359, no. 20, pp. 2095-2104, 2008.

[4] Y. S. Chiew, C. Pretty, P. D. Docherty, B. Lambermont, G. M. Shaw, T. Desaive, and J. G. Chase, "Time-Varying Respiratory System Elastance: A Physiological Model for Patients Who Are Spontaneously Breathing," PLoS One, vol. 10, no. 1, p. e0114847, 2015.

[5] E. Akoumianaki, A. Lyazidi, N. Rey, D. Matamis, N. Perez-Martinez, R. Giraud, J. Mancebo, L. Brochard, and J.-C. Marie Richard, "Mechanical ventilation-induced reverse-triggered breaths: a frequently unrecog- nized form of neuromechanical coupling." Chest, vol. 143, no. 4, pp. 927-38, Apr. 2013.

[6] J. H. Bates, Lung mechanics: an inverse modeling approach. New York: Cambridge University Press, 2009.

[7] P. D. Docherty, C. Schranz, Y.-S. Chiew, K. Möller, and J. G. Chase, "Reformulation of the pressure-dependent recruitment model (PRM) of respiratory mechanics," Biomed. Signal Process. Control, vol. 12, pp. 47-53, Jul. 2014.

[8] A. Szlavecz, Y. Chiew, D. Redmond, A. Beatson, D. Glassenbury, S. Corbett, V. Major, C. Pretty, G. M. Shaw, B. Benyo, T. Desaive, and J. Chase, "The Clinical Utilisation of Respiratory Elastance Software (CURE Soft): a bedside software for real-time respiratory mechanics monitoring and mechanical ventilation management," Biomed. Eng. Online, vol. 13, no. 1, p. 140, 2014.

[9] C. Schranz, P. D. Docherty, Y. S. Chiew, K. Möller, and J. G. Chase, "Iterative integral parameter identification of a respiratory mechanics model," Biomed. Eng. Online, vol. 11, no. 38, pp. 1-14, 2012.

[10] C. Schranz, C. Knöbel, J. Kretschmer, Z. Zhao, and K. Möller, "Hierarchical parameter identification in models of respiratory mechanics." IEEE Trans. Biomed. Eng., vol. 58, no. 11, pp. 3234-41, Nov. 2011.

[11] Z. Zhao, J. Guttmann, and K. Möller, "Assessment of a volumedependent dynamic respiratory system compliance in ALI/ARDS by pooling breathing cycles," Physiol. Meas., vol. 33, no. 8, pp. N61-N67, 2012.

[12] J. Beck, S. B. Gottfried, P. Navalesi, Y. Skrobik, N. Comtois, M. Rossini, and C. Sinderby, "Electrical activity of the diaphragm during pressure support ventilation in acute respiratory failure." Am. J. Respir. Crit. Care Med., vol. 164, no. 3, pp. 419-24, Aug. 2001.

[13] D. H. Rhoney and K. R. Murry, "National survey of the use of sedating drugs, neuromuscular blocking agents, and reversal agents in the intensive care unit." J. Intensive Care Med., vol. 18, no. 3, pp. 13945, 2003. 